\title{
Epidemiology and organization of care in medically unexplained symptoms: a systematic review with a focus on cultural diversity and migrants
}

\author{
Peter Vermeir ${ }^{1}$, An Mariman ${ }^{2}$, Lilla Lucza ${ }^{3}$, Sallay Viola ${ }^{3}$, Anne Weiland ${ }^{4}$, Karen \\ Stegers-Jager ${ }^{5}$, and Vogelaers Dirk ${ }^{1}$ \\ ${ }^{1}$ Ghent University Hospital \\ ${ }^{2}$ Ghent University Hospital \\ ${ }^{3}$ University of Szeged Faculty of Medicine \\ ${ }^{4}$ Erasmus University Rotterdam \\ ${ }^{5}$ Erasmus Universiteit Rotterdam
}

January 3, 2021

\begin{abstract}
Background: Since the pathophysiology of medically unexplained symptoms (MUS) remains unclear, health care providers often struggle with these patients, especially with a different ethnic and/or cultural background. These challenges are insufficiently addressed in their training and in the organization of care. Aim: To improve health care provider-patient interaction focused on MUS patients in general and in ethnic minorities and refugees in particular through a systematic review of syndromal definitions and epidemiology and organization of care of MUS patients. Methods: Screening of PubMed, Web of Science, Cinahl and Cochrane Library on the keywords 'Medical unexplained (physical) symptoms (MUPS)', 'Somatoform disorder', 'Functional syndrome', 'Diversity', 'Migrants', 'Ethnicity', 'Care models', 'Medical education', 'Communication skills', 'Health literacy' Results: Different case definitions result in markedly different epidemiologic estimates for MUS patients. Nevertheless, they are prevalent in a wide range of health care settings. Literature offers evidence of the effectiveness of structural frameworks in approaching MUS patients. Organization of MUS care needs to transcend different levels of care: specialist tertiary and secondary care and primary care involving different qualifications of caregivers need to be aligned. Conclusion: The systematic review identified significant gaps and shortcomings in organization of care. These need to be addressed in order to improve outcomes. Keywords: Medical unexplained (physical) symptoms (MUPS), Somatoform disorder, Functional syndrome, Diversity, Migrants, Ethnicity, Care models Message for the clinic: - Medically unexplained symptoms are highly prevalent but case definitions hamper both recognition and a proper approach. - MUS are ill understood in both the general population as in migrants and refugees. - Interdisciplinary and integrated care through a biopsychosocial model is mandatory.
\end{abstract}

Epidemiology and organization of care in medically unexplained symptoms: a systematic review with a focus on cultural diversity and migrants

Running title - MUS: a care challenge in a multicultural society

An Mariman

Ghent University/Ghent University Hospital

Peter Vermeir

Ghent University / Ghent University Hospital 
Lilla Lucza

University of Szeged

Viola Sallay

University of Szeged

Anne Weiland

Erasmus MC University Medical Center, Rotterdam, The Netherlands

Karen M. Stegers-Jager

Erasmus MC University of Rotterdam, The Netherlands

Dirk Vogelaers

Ghent University/ Ghent University Hospital

Note: The two first author equally contributed to the work.

Corresponding author:

Peter Vermeir

Ghent University/ Ghent University Hospital

C.Heymanslaan 10

9000 Ghent (Belgium)

$+3293322349$

Acknowledgment

The study was funded by the European Comission, Erasmus+ Strategic Partnership aimed at Medical Education on Medically Unexplained Symptoms and Intercultural Communication. /MUSIC/ Nr. 20182021.2018-1-NL01_KA203-038971

\section{ABSTRACT}

\section{Background:}

Since the pathophysiology of medically unexplained symptoms (MUS) remains unclear, health care providers often struggle with these patients, especially with a different ethnic and/or cultural background. These challenges are insufficiently addressed in their training and in the organization of care.

Aim:

To improve health care provider-patient interaction focused on MUS patients in general and in ethnic minorities and refugees in particular through a systematic review of syndromal definitions and epidemiology and organization of care of MUS patients.

\section{Methods:}

Screening of PubMed, Web of Science, Cinahl and Cochrane Library on the keywords 'Medical unexplained (physical) symptoms (MUPS)', 'Somatoform disorder', 'Functional syndrome', 'Diversity', 'Migrants', 'Ethnicity', 'Care models', 'Medical education', 'Communication skills', 'Health literacy'

\section{Results:}

Different case definitions result in markedly different epidemiologic estimates for MUS patients. Nevertheless, they are prevalent in a wide range of health care settings. Literature offers evidence of the effectiveness of structural frameworks in approaching MUS patients. Organization of MUS care needs to transcend different 
levels of care: specialist tertiary and secondary care and primary care involving different qualifications of caregivers need to be aligned.

Conclusion:

The systematic review identified significant gaps and shortcomings in organization of care. These need to be addressed in order to improve outcomes.

Keywords: Medical unexplained (physical) symptoms (MUPS), Somatoform disorder, Functional syndrome, Diversity, Migrants, Ethnicity, Care models

Message for the clinic:

- Medically unexplained symptoms are highly prevalent but case definitions hamper both recognition and a proper approach.

- MUS are ill understood in both the general population as in migrants and refugees.

- Interdisciplinary and integrated care through a biopsychosocial model is mandatory.

Introduction

Both in primary and in specialist care, caregivers frequently have to deal with or feel even confronted with patients consulting with medically unexplained (physical) symptoms (MUS). In the literature MUS prevalences vary between $30-50 \%[17,35,36]$. MUS are categorized into syndromes without robust evidence for these classifications. They can be subdivided on the basis of symptom duration, number of symptoms and their impact on life quality and daytime functioning into mild, moderately-severe and severe.

Although the pathophysiology of MUS remains unclear, theoretical explanatory frameworks combine and integrate biological (physiological), psychological and social factors (e.g. in the biopsychosocial model, the stress-vulnerability model, stress model, the perceptual-cognitive model, the neurobiological model, vicious circles and emotions with references in sequence of the mentioned models [3, 10, 11, 12, 18, 28]. All models are only to a limited extent based on empiric research. Complementary to using an acceptable explanatory model, the quality of communication is crucial in the health care provider-patient relationship. In general and in particular in the setting of MUS, the quality of this relationship and of the mutual communication has a positive impact on health outcomes, patient satisfaction and therapeutic adherence $[1,13]$. MUS patients often feel not-understood and many health care providers experience feelings of helplessness, potentially leading to frustrations and irritation on both sides and limiting the required patient-centeredness. This may trigger repeated consultations and increased consumption of resources, and provoke medical shopping.

Increased use of healthcare resources is even more frequent in patients with a different ethnic background [34]. Moreover the healthcare sector is challenged to deal with the current issues of migration and the presence of large groups of refugees in the European Union in a positive way. Not only patients with immigrant backgrounds, but also racial, ethnic, and sexual minorities experience disparities in access to healthcare, quality of care received, and caregiver services [7].

A positive approach often is lacking in the organization of care for MUS patients, of whatever cultural and ethnic background. This systematic review therefore aims at a critical synthesis of the current literature on the predefined domains of, first, syndromal definitions and epidemiology of MUS; second, organization of care for MUS patients, in order to define gaps as well as areas for improvement and derive recommendations.

Methods

A systematic search was carried out on the databases PubMed, Web of Science, Cinahl and The Cochrane Library using the keywords: 'Medically unexplained (physical) symptoms (MUS)', 'Somatoform disorder', 'Functional syndrome', 'Diversity', 'Migrants', 'Ethnicity', 'Care models', 'Medical education', 'Communication skills', 'Health literacy'. The keywords were internally validated by the co-authors. A framework with two categories was predefined: syndromal definitions and epidemiology of MUS and organization of care. In 
order to qualify for this review articles needed to be 1) published between January 1, 2002 and September 30, 2019, 2) available as full text in English 3) categorizable as original research, reviews, meta-analyses or letters to the editor. Database screening was closed $2^{\text {nd }}$ of October 2019. Only articles in the English language were included in order to avoid misinterpretations. Titles and abstracts were reviewed to verify inclusion criteria. If all inclusion criteria were present or if this remained unclear, the articles were fully read. All studies were screened for eligibility by two independent reviewers (PV, AM) who reviewed titles, abstracts and full text. Any disagreements were resolved by discussion and, if necessary a third reviewer (DV) was consulted. Additional literature was obtained through searching references in the manuscripts (snowball method).

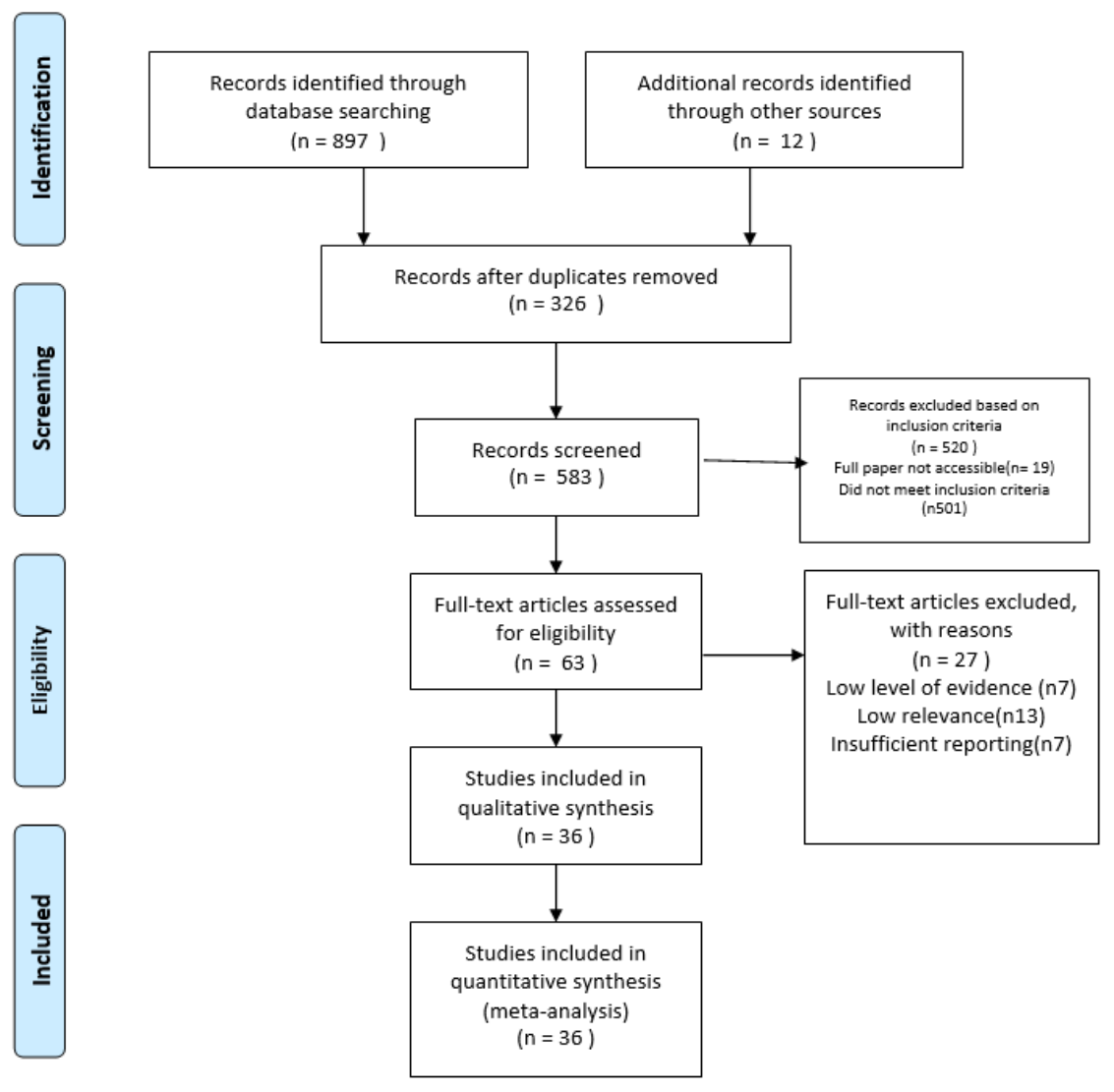

Figure 1: Review stages based on PRISMA flow diagram

The results of the search process are summarized into a PRISMA flow diagram (Figure 1). Out of a total of 909 papers selected, 326 duplicates were removed. After screening the search in PubMed yielded 278, in Web of Science 302, in Cinahl 158 and in the Cochrane Library 159 articles. Through the snowballing method of screening the reference lists of relevant articles, 12 additional articles complying with the inclusion criteria could be identified and were added.

After screening 583 papers on title and abstract 63 papers remained for full-text screening. From these, 36 articles were subjected to quality assessment.

\section{Syndromal definitions and epidemiology of MUS}

Insights and recognition of the prevalence, impact and the relativity of the definitions used in current literature is an important prerequisite for health care providers in their management of MUS. Indeed, the 
prevalence of MUS is highly dependent on the case definitions used. All definitions are syndromal and have the character of a checklist approach. This is illustrated by the classification of a multi-setting population of patients with MUS into either DSM IV somatization (undifferentiated somatoform and pain) disorder versus DSM V somatic symptom disorder (SDD) [6]. In a questionnaire study involving baseline data of 325 MUS patients in $1^{\text {st }}, 2^{\text {nd }}$ and $3^{\text {rd }}$ lines of health care, DSM V SSD criteria proved more restrictive, as only $45.5 \%$ fulfilled these criteria while $92.9 \%$ of participants were included using the DSM IV criteria. DSM V SSD and the presence of psychologic criteria selected for more symptom severity and physical dysfunction in MUS. With chronification the semantic discussion remains, as exemplified in the review of De Gucht et al. 2002, proposing a distinction into presenting somatization secondary to psychological distress versus a primary phenomenon characterized by the presence of MUS (functional somatization). More recently, literature has moved away from this labeling approach and focused on functional limitations and possibly on different phenotypes that may be relevant for differential effects of illness on disability. Furthermore there is considerable overlap between the definitions of the different functional somatic syndromes [23, 24].

Whatever the limitations and the semantics of syndromal definitions, patients frequently present in primary care with multiple somatic complaints, that can be associated with significant distress and functional impairment. These can become chronic in 20 to $25 \%$ of cases, associated with high use of medical services and increased risk of iatrogenic complications [9]. In a point prevalence primary care study, GP's assessed presenting complaints as unexplained in $13 \%$ of consecutive at random consultations [35]. In a large scale questionnaire survey of morbidity in 400.000 patients aged 18 years and older and visiting their GP at least once a year in 104 general practices in the Netherlands, 25-50\% of all reasons for a GP visit concerned medically unexplained symptoms, $20 \%$ of GP-patient contacts physical symptoms without a pathological explanation. However, the frequent presentation of such symptoms within the same individual is significantly rarer: only $2.45 \%$ presented at least 4 times in a single year period with symptoms considered medically unexplained and lacking a medical diagnosis. Socio-demographically these patients were significantly older, more often female, less educated, more frequently unemployed and more frequently of a non-Western origin than "average" patients or patients with a medical diagnosis [36].

These patients also represent a considerable burden in acute care. In a retrospective chart study of 2869 adult patients at three tertiary care emergency departments (ED) MUS were present in $13.4 \%$ of all ED admissions, and in $18.5 \%$ of patients, after exclusion of trauma. Patients with MUS on the ED were more often younger, female, self-referred and frequent visitors and more often had psychiatric disease. Dutch and Belgian hospitals differed in the distribution of patients in triage categories and the incidence of psychiatric illnesses. Hence, patients with MUS represent a major burden to ED's, as in primary and secondary healthcare [2]. In an older retrospective cohort study in secondary care, the proportion of MUS in frequent attenders represented $54 \%$ in gastroenterology, $50 \%$ in neurology, $34 \%$ in cardiology, $33 \%$ in rheumatology, $30 \%$ in orthopedic clinics, $27 \%$ in otorhinolaryngology and even $17 \%$ in general surgery and gynecology and $15 \%$ in pulmonary medicine [27]. Hence, they represent a significant segment of medical activity and are transversally relevant in most domains. A cross-sectional survey in two London general hospitals estimated the prevalence and risk factors for MUS across a variety of specialties; about half of the respondents from seven outpatient clinics fulfilled criteria for MUS, representing the most common diagnosis in some specialties with the highest prevalence in the gynaecology clinic $(66 \%)$. After adjustment for confounders, MUS were associated with female gender, younger age and current employment. Psychiatric comorbidity was not associated per se with the presence of MUS but was more likely in patients with multiple symptoms [23, 24].

In a review on somatization in refugees in non-Western countries [29] the prevalence of somatization disorder was shown to be equal in different refugee populations world-wide. The number of MUS is generally higher among refugees as compared to non-refugees, but studies are not comparable, as different definitions of somatization were used, such as in clinical assessments and conclusions from questionnaires. Somatization in refugees is strongly connected with psychopathology and possibly with traumatization and with negative life events and might be perceived as a specific idiom of distress, which accompanies PTSD. Hence, refugee populations require specific screening for clinical PTSD. Kounou et al, 2017 explored the relationship between peritraumatic reactions, PTSD symptoms, somatization and quality of life in a retrospective, cross-sectional 
study of a large sample of 101 adult refugees ( $45 \%$ of the female gender) 2 years after the 2011 Ivory Coast sociopolitical crisis. They demonstrated that $86,1 \%$ of participants scored above the threshold for probable clinical PTSD. Peritraumatic dissociation and stress were significantly associated with PTSD symptoms. Somatization and PTSD symptoms negatively correlated with quality of life. [19].

\section{Organization of care for MUS patients}

Bestall et al (2017) described the process of setting up and the early results of a novel liaison psychiatry service in primary care for people identified as frequent general practice attenders with long-term conditions or MUS. They retrospectively extracted data from the EHR on patient reported outcome and experience measures in a limited number of 19 patients and caregiver experience. The expert group developing this program acknowledged the need for long term and individualized care, translating in the need for a "patient" patient approach. Calling for this patience and longer term management goals may run against the grain of modern physicians and society geared towards quick and short term results. This should reflect in attitudes towards MUS patients.

This need for individualization was also substantiated by a narrative review of the literature between 1985 and 2000 on published evidence relevant to primary care dealing with a culturally diverse, challenging MUS patient population. No single approach will effectively treat all patients with MUS in primary care. Patient-centeredness and attention to biopsychosocial needs, patience and empathic communication are core characteristics. Patient-centered care can be the key to cultural competence too. A newer, cross cultural patient centered approach focuses on foundational communication skills, awareness of cross-cutting cultural and social issues, and health beliefs that are present in all cultures. This approach relies on identifying and negotiating different styles of communication, decision-making preferences, sexual and gender issues, roles of family, and issues of mistrust, prejudice, and racism, among other factors. They describe 'cultural' challenges that arise in the care of four patients from disparate cultures, then illustrate how to apply principles of patient centered care to these arising challenges [15].

Clinical assessment of MUS patients should include psychological concerns, family and cultural issues, screening for a history of a dysfunctional childhood and for symptoms of depression, anxiety and PTSD. This can be followed by confirmation that symptoms are real, even when linked to psychosocial stress [14] and an individualized treatment, including elements of reattribution, progressive muscle relaxation and related techniques, group or individual CBT, by either GP or mental health clinician) and medication. If indicated for depressive illness, antidepressants should be fully dosed, in insomnia low dose antidepressant medications (antidepressants with sedative properties) should be considered, while avoiding benzodiazepines. The time taken to develop one's own clinical approach can contribute significantly to patient welfare and their family members, as well as to the GP's own personal and professional growth [14].

Brownell et al (2016) presented an interim practical management guide (IPMG) that clinical practitioners may find useful. This framework was deduced from or based upon interview data of 12 family physicians and 18 specialists from different domains in two urban centers in 2 different Canadian provinces. Four key themes emerged from the interviews, namely the challenge of diagnosis, the challenge of management and treatment, the importance of communication and, finally, the importance of therapeutic relationship. Key points include the early consideration of a diagnosis of MUS, the limitation of investigations to essentials, a clear definition of the physician responsible for provision of clinical follow up, the assurance by this physician of ongoing patient commitment to care even if a diagnosis is not forthcoming, the development of a care framework for the patient to follow, including supportive lifestyle approaches, the education of the patient about MUS and the distinction between "being resigned to their fate" and commitment to managing symptoms while enhancing life quality, avoidance of exposing the patient to harmful treatments and invasive investigations and a focus on the importance of communication. The key point is to provide care within a framework. This framework should also allow sufficient contact time in order to address uncertainty and complexity in MUS patients. In the in-depth interviews of junior doctors in the UK gaps in service delivery were acknowledged and included insufficient time and resources to explore patient's needs [37]. 
In a narrative review with management recommendations Croicu et al (2014) focused on the approach of patients with multiple and persistent physical symptoms, that commonly present in primary care. They stressed the need for collaboration with the patient for effective engagement setting treatment goals in a model of shared decision making. They also indicated screening and treatment of depression and anxiety disorders as a key component of management and that patients should be educated about how psychosocial stressors and somatic symptoms interact. Medically unexplained, persistent or multiple somatic symptoms should heighten a physician's clinical suspicion of a co-morbid or potentially treatable depressive or anxiety disorder: other predictors of depression or anxiety include recent $\mathbf{S}$ tress, poor Self rated health, high Symptom Severity (conceptualized in a $4 \mathrm{~S}$ model), perception by the health care provider of a difficult patient encounter, repeated clinic visits and other chronic somatic symptoms [20]. Providers should avoid setting up a dichotomy between mental and physical causation of symptoms.

Olde Hartman et al (2017) addressed current problems in the management of undifferentiated MUS in primary care in a narrative review, encompassing guidelines and systematic reviews. They indicated the limited evidence base of the recommendations and several barriers to their implementation. More evidence is needed on the effect of strengthening the doctor-patient relationship on the course and prognosis of MUS, the influence of specific consultation skills, the effects of physical therapy and ways to deliver psychological treatment more effectively in primary care of patients with MUS. As an example, training GP's to deliver brief reattribution type interventions in routine consultations improves their skills and attitudes but evidence of improved patient outcomes is lacking. Improving GP explanation of unexplained symptoms proved insufficient to reduce patient's concerns in a thematic analysis of in depth interviews comparing a trained vs a control GP group. Patient's decisions over how much, and what information they present to GPs limits the effectiveness of communication training [26]. Hence, Olde Hartman et al (2017) advocate that severe and complex cases should be managed in collaboration with specialists in a stepped-care approach, as psychological treatment delivered by specialists, especially CBT, has the strongest evidence for patient benefit.

Cooper et al (2017) provided an outline of the development and implementation of an ISTDP (Intensive Short Term Dynamic Psychotherapy) service for MUS across two community-based academic family medicine teaching clinics. Preliminary clinical and cost outcome data gathered over the service's first 18 months were reported: patients reported significantly decreased somatic symptoms in the Patient Health Questionnaire15 , while family physicians' visits as a proxy to medical consumption decreased in the six months follow after attending the MUS service in comparison to the 6 months prior and both patients and primary care physicians reported a high degree of satisfaction with the service. Assessment of MUS and functional syndromes requires a timely and thorough diagnostic process, in which the biopsychosocial model needs to be the framework but also including somatic reassurance. An example of such an integrated multidisciplinary path was described by Tobback et al (2017) and consists of an as simultaneous as possible somatic, rehabilitation, psychological (including psychodiagnostic testing) and sleep and sleepiness (through polysomnography and multiple sleep latency testing) assessment. This was followed by multidisciplinary discussion, leading to (an often composite) diagnosis and individualized treatment proposal. The concept also stresses the need for interaction with primary care, not in the least in order to allow early detection of somatoform disorders, in a stage that these may be more prone to change and improvement. This approach allowed for more differentiated and multifaceted diagnostication for patients referred with chronic fatigue and presumed chronic fatigue syndrome, identifying an array of CFS without comorbidity, comorbid sleep and psychiatric disorders and finally, previously unrecognized or unacknowledged primary sleep and/or psychiatric disorders, that warrant separate approaches [22].

Ryckegem et al (2017) explored the experiences and expectations of patients with CFS, a major MUS presentation, that were assessed in a dedicated tertiary care referral center for CFS and their general practitioners in semi-structured interviews using open explorative thematic coding. Ambiguities about CFS resulted in mixed feelings in patients, caregivers and the patient environment. A clear need for punctuality, continuity and relevance of reporting as well as of information and education, which also included a clear model of explanation, was identified. There is a need for structured information about the diagnostic process, e.g. 
through information leaflets. A central intermediator, which is often lacking in the effective implementation of the therapeutic program, seemed essential in order to address gaps in coordination of care.

Attention to diversity issues should be considered during all stages of health care planning, including recruiting and training of health-care staff and organizing and providing health care [31]. The characteristics of in-hospital care and treatment of immigrant patients were explored in semi-structured interviews with care providers regarding ethnic disparities in patient safety [32]. These patient safety events occur through insufficient acquisition in cultural competence (e.g cultural knowledge, attitudes, skills and resources) and are not merely due to ethnic considerations. Medical residents found it easier to "get by" without a professional interpreter even though they were aware of negative implications for quality of care. This was not only driven by time constraints or lack of availability but also by morality and cultural competence. Three key patterns were identified. Patient safety events occur because of a) inappropriate response by health care providers to objective characteristics in immigrant patients, such as low language proficiency, lack of health insurance or genetic conditions; b) misunderstandings between patients and care providers because of differences in illness perceptions and expectations about care and treatment, and c) inappropriate care because of providers' prejudices against or stereotypical ideas regarding immigrant patients [32].

Discussion and recommendations

It seems important to recognize that diagnostic criteria used in the field of MUS are merely syndromal descriptions. This trend towards diagnostic reductionism which translates into labels such as chronic fatigue syndrome, fibromyalgia, DSM IV somatization disorder or DSM 5 somatic symptom disorder, needs to be superseded or at least complemented by an individual approach. Recognition of symptoms and the dimensions of illness and disability is more important than a debatable and debated diagnosis in search of a disease. This complexity in which cause and effect relationships are not clear and cause and effect seem to merge as they influence each other, as in the interaction between fatigue and pain, needs to be recognized and translated by the caregiver in the approach and communication of MUS patients. It may prove counterproductive to be too restrictive in the case definitions, in particular if the case definition tends to select for more severe cases, that may be less flexible to change.

In a descriptive review and editorial comment cultural and socioeconomic factors proved powerful predictors of individual somatic symptom perception and health care utilization in the domain of functional neurologic syndromes [21]. Dualistic health care systems with separation between somatic and mental health disciplines produce delayed diagnoses (with a mean estimated duration between onset of somatoform disorder and first psychotherapeutic and psychiatric treatment of 25 years) and increase stigma for mental disorders. They stress the need to include available and validated self-report instruments for screening and early diagnosis of functional disorders and somatic symptom disorders (Somatic Symptom Disorder B-criteria scale-12, Patient Health Questionnaire-15). The European Network to improve diagnosis, treatment and health care in patients with persistent somatic symptoms (EURONET-SOMA) published recommendations for core outcome domains in the evaluation of interventions. Early recognition and treatment prevent unnecessary suffering and inappropriate health care utilization. The approach of functional disorders requires explanatory models for the pathway from symptom perception to functional syndromes. Access to effective diagnosis and treatment for all patients, accounting for cultural background, an emphasis on patient empowerment and early participation in the treatment process are key to outcome improvements. This implies enhancements in interdisciplinary training and collaboration between somatic and mental health disciplines.

Future interventions in the communication between even trained GPs and their patients need to help patients to make sense of the complex nature of their problems, reassure that medical attention to psychosocial factors does not preclude vigilance to physical disease and establish a quality of relationship in which patients do not perceive psychosocial enquiry as inappropriate and that fosters an environment in which physicians can support patient self-management [26].

Literature offers evidence of the effectiveness of structural frameworks in approaching MUS patients [5, 8, 33]. 
Organization of MUS care needs to transcend different levels of care: specialist tertiary and secondary care and primary care involving different qualifications of caregivers need to be aligned. This is essential as in present day Descartian-inspired Western society most patients expect simple biomedical explanations for their unexplained symptoms and struggle, as well as their caregivers, with the complexity of the bio-psychosocial explanation. This includes drifting away from e.g a diagnosis of primary or co-morbid psychiatric conditions. This stresses the need for communication between all actors around the patient and substantiates the need for coordination, with a potential role of dedicated advanced nurse practitioners or case managers, providing the necessary glue in often fragmented and insufficiently interactive care systems, lacking integration. Optimal use of electronic carriers and platforms of information can enhance the integration of the roles of all health care providers involved, in order to ensure continuity and sharing of common views and strategies. The use of these communication tools requires the solution of specific technical and organizational issues and also specific training.

Research should focus on how the implementation of such a function of coordination can increase patient adherence, satisfaction and health-related quality of life and facilitate effective cooperation between the involved parties, to increase cost-effectiveness and the likelihood of desired outcomes of the diagnostic and therapeutic process [30]. Finally, expectations need to be tailored to realistic objectives, which often proves difficult in MUS patients. This again underscores the need for structured and longitudinal follow-up, coaching and encouraging patients along the long track to improvement. It should be recommended, as society as well as the medical community feels these patients are not prone to improvement of quality of life or socioprofessional reintegration, to prove the effectiveness of structured long-term follow up by health care professionals adequately trained in the field through relevant outcome measures (PROM and PREM). These should be included as a tool in follow up consultations; their use should not restricted to the generation of service statistics.

acknowledgments

The study was funded by the European Commission, Erasmus+ Strategic Partnership aimed at Medical Education on Medically Unexplained Symptoms and Intercultural Communication. /MUSIC/ Nr. 20182021.2018-1-NL01_KA203-038971

\section{References}

1. Aiarzaguena, J. M., Grandes, G., Gaminde, I., Salazar, A., SANchez, A., \& AriNO, J. (2007). A randomized controlled clinical trial of a psychosocial and communication intervention carried out by GPs for patients with medically unexplained symptoms. Psychological medicine, 37(2), 283-294.

2. Alsma, J., van de Wouw, J., Jellema, K., Coffeng, S. M., Tobback, E., Delesie, L., van den Brand, C. L., et al. (2019). Medically unexplained physical symptoms in patients visiting the emergency department : an international multicentre retrospective study. EUROPEAN JOURNAL OF EMERGENCY MEDICINE.

3. Bankier, B., Aigner, M., \& Bach, M. (2001). Alexithymia in DSM-IV disorder: comparative evaluation of somatoform disorder, panic disorder, obsessive-compulsive disorder, and depression. Psychosomatics, 42(3), 235-240.

4. Bestall, J., Siddiqi, N., Heywood-Everett, S., Freeman, C., Carder, P., James, M., .. \& House, A. (2017). New models of care: a liaison psychiatry service for medically unexplained symptoms and frequent attenders in primary care. BJPsych bulletin, 41(6), 340-344.

5. Brownell, A. K. W., Atkins, C., Whiteley, A., Woollard, R. F., \& Kornelsen, J. (2016). Clinical practitioners' views on the management of patients with medically unexplained physical symptoms (MUS): a qualitative study. BMJ open, 6(12), e012379.

6. Claassen-van Dessel, N., van der Wouden, J. C., Dekker, J., \& van der Horst, H. E. (2016). Clinical value of DSM IV and DSM 5 criteria for diagnosing the most prevalent somatoform disorders in patients with medically unexplained physical symptoms (MUPS). Journal of psychosomatic research, 82, 4-10.

7. Collins, J. C., \& Rocco, T. S. (2014). Disparities in healthcare for racial, ethnic, and sexual minorities. New Directions for Adult and Continuing Education, 2014(142), 5-14. 
8. Cooper, A., Abbass, A., Zed, J., Bedford, L., Sampalli, T., \& Town, J. (2017). Implementing a psychotherapy service for medically unexplained symptoms in a primary care setting. Journal of clinical medicine, 6(12), 109.

9. Croicu, C., Chwastiak, L., \& Katon, W. (2014). Approach to the patient with multiple somatic symptoms. Medical Clinics, 98(5), 1079-1095.

10. Dantzer, R. (2005). Somatization: a psychoneuroimmune perspective. Psychoneuroendocrinology, 30(10), 947-952.

11. De Gucht, V., \& Fischler, B. (2002). Somatization: a critical review of conceptual and methodological issues. Psychosomatics, 43(1), 1-9.

12. De Gucht, V., Fischler, B., \& Heiser, W. (2004). Neuroticism, alexithymia, negative affect, and positive affect as determinants of medically unexplained symptoms. Personality and Individual Differences, 36(7), 1655-1667.

13. Dobkin, P. L., De Civita, M., Abrahamowicz, M., Baron, M., \& Bernatsky, S. (2006). Predictors of health status in women with fibromyalgia: a prospective study. International journal of behavioral medicine, 13(2), 101-108.

14. Edwards, T. M., Stern, A., Clarke, D. D., Ivbijaro, G., \& Kasney, L. M. (2010). The treatment of patients with medically unexplained symptoms in primary care: a review of the literature. Mental Health in Family Medicine, 7(4), 209.

15. Epner, D. E., \& Baile, W. F. (2012). Patient-centered care: the key to cultural competence. Annals of oncology, 23(suppl_3), 33-42.

16. Hawker, S., Payne, S., Kerr, C., Hardey, M., \& Powell, J. (2002). Appraising the evidence: reviewing disparate data systematically. Qualitative health research, 12(9), 1284-1299.

17. Khan, A. A., Khan, A., Harezlak, J., Tu, W., \& Kroenke, K. (2003). Somatic symptoms in primary care: etiology and outcome. Psychosomatics, 44(6), 471-478.

18. Kirmayer, L. J., \& Looper, K. J. (2006). Abnormal illness behaviour: physiological, psychological and social dimensions of coping with distress. Current Opinion in Psychiatry, 19(1), 54-60.

19. Kounou, K. B., Brodard, F., Gnassingbe, A., Dogbe Foli, A. A., Sager, J. C., Schmitt, L., \& Bui, E. (2017). Posttraumatic Stress, Somatization, and Quality of Life Among Ivorian Refugees. Journal of traumatic stress, 30(6), 682-689.

20. Kroenke, K. (2003). Patients presenting with somatic complaints: epidemiology, psychiatric comorbidity and management. International journal of methods in psychiatric research, 12(1), 34-43.

21. Löwe, B., \& Gerloff, C. (2018). Functional somatic symptoms across cultures: perceptual and health care issues. Psychosomatic medicine, 80(5), 412-415.

22. Mariman, A., Delesie, L., Tobback, E., Hanoulle, I., Sermijn, E., Vermeir, P., . . \& Vogelaers, D. (2013). Undiagnosed and comorbid disorders in patients with presumed chronic fatigue syndrome. Journal of psychosomatic research, 75(5), 491-496.

23. Nimnuan, C., Hotopf, M., \& Wessely, S. (2001). Medically unexplained symptoms: an epidemiological study in seven specialities. Journal of psychosomatic research, 51(1), 361-367.

24. Nimnuan, C., Rabe-Hesketh, S., Wessely, S., \& Hotopf, M. (2001). How many functional somatic syndromes?. Journal of psychosomatic research, 51(4), 549-557.

25. Olde Hartman, T. C., Rosendal, M., Aamland, A., van der Horst, H. E., Rosmalen, J. G., Burton, C. D., \& Lucassen, P. L. (2017). What do guidelines and systematic reviews tell us about the management of medically unexplained symptoms in primary care?. Br J Gen Pract Open, 1(3), bjgpopen17X101061.

26. Peters, S., Rogers, A., Salmon, P., Gask, L., Dowrick, C., Towey, M., .. \& Morriss, R. (2009). What do patients choose to tell their doctors? Qualitative analysis of potential barriers to reattributing medically unexplained symptoms. Journal of general internal medicine, 24(4), 443.

27. Reid, S., Wessely, S., Crayford, T., \& Hotopf, M. (2001). Medically unexplained symptoms in frequent attenders of secondary health care: retrospective cohort study. Bmj, 322(7289), 767.

28. Rief, W., \& Broadbent, E. (2007). Explaining medically unexplained symptoms-models and mechanisms. Clinical psychology review, 27(7), 821-841.

29. Rohlof, H. G., Knipscheer, J. W., \& Kleber, R. J. (2014). Somatization in refugees: a review. Social 
psychiatry and psychiatric epidemiology, 49(11), 1793-1804.

30. Ryckeghem, H., Delesie, L., Tobback, E., Lievens, S., Vogelaers, D., \& Mariman, A. (2017). Exploring the potential role of the advanced nurse practitioner within a care path for patients with chronic fatigue syndrome. Journal of advanced nursing, 73(7), 1610-1619.

31. Sorensen, J., Norredam, M., Dogra, N., Essink-Bot, M. L., Suurmond, J., \& Krasnik, A. (2017). Enhancing cultural competence in medical education. International journal of medical education, 8, 28.

32. Suurmond, J., Uiters, E., de Bruijne, M. C., Stronks, K., \& Essink-Bot, M. L. (2010). Explaining ethnic disparities in patient safety: a qualitative analysis. American journal of public health, 100(S1), S113-S117.

33. Tobback, E., Mariman, A., Heytens, S., Declercq, T., Bouwen, A., Spooren, D., .. \& Vogelaers, D. (2014). A multidisciplinary network for the care of abnormal fatigue and chronic fatigue syndrome in the provinces of East and West Flanders in Belgium. Acta Clinica Belgica, 69(5), 327-334.

34. Van der Lucht, F., \& Verweij, A. (2010). Etniciteit en zorggebruik. Nationaal Kompas Volksgezondheid. Bilthoven: RIVM.

35. van der Weijden, T., van Velsen, M., Dinant, G. J., van Hasselt, C. M., \& Grol, R. (2003). Unexplained complaints in general practice: prevalence, patients' expectations, and professionals' test-ordering behavior. Medical Decision Making, 23(3), 226-231.

36. Verhaak, P. F., Meijer, S. A., Visser, A. P., \& Wolters, G. (2006). Persistent presentation of medically unexplained symptoms in general practice. Family Practice, 23(4), 414-420.

37. Yon, K., Nettleton, S., Walters, K., Lamahewa, K., \& Buszewicz, M. (2015). Junior doctors' experiences of managing patients with medically unexplained symptoms: a qualitative study. BMJ open, 5(12), e009593.

\section{Hosted file}

PRISMA flow diagram.pdf available at https://authorea.com/users/386884/articles/502287epidemiology-and-organization-of-care-in-medically-unexplained-symptoms-a-systematicreview-with-a-focus-on-cultural-diversity-and-migrants 